\title{
Introduction to Special Issue on Terahertz Metrology
}

\author{
Thomas Kleine-Ostmann
}

Received: 8 May 2014 / Accepted: 12 May 2014/

Published online: 18 May 2014

(C) Springer Science+Business Media New York 2014

Metrology is the art and science of measurement. Precise and reliable measurement methods leading to trustable measurement results with known measurement uncertainty are a prerequisite for meaningful scientific results, comparability and interoperability in a multitude of disciplines in physics and technology. Implementing traceability to the International System of Units (Système international d'unités - SI) by establishing an unbroken chain of calibrations to the representation of fundamental or derived units at the National Metrology Institutes (NMIs) is the basis for this.

With the advent of new measurement techniques in THz science and technology, metrology aspects become more important in this emerging field as commercial $\mathrm{THz}$ systems are appearing on the market. E.g., the compliance with safety limits cannot be proven and interoperability of system components cannot be guaranteed without reliable measurement results including the measurement uncertainty. NMIs but also many other scientific groups work on open questions in the field of THz metrology such as detector calibration, characterization of ultrafast devices, traceability for scattering parameters in vector network analysis and measurement uncertainty in $\mathrm{THz}$ spectroscopy.

In this special issue on $\mathrm{THz}$ Metrology seven invited articles cover the most recent developments in the field. The articles are meant to give a review of the respective topic combined with the most recent results from the authors.

1. Terahertz frequency combs: Theoretical aspects and applications by Heiko Füser and Mark Bieler from Physikalisch-Technische Bundesanstalt, the German NMI in Braunschweig, introduces the $\mathrm{THz}$ frequency comb as a metrological tool and shows how it is used by the authors for high precision frequency measurements over a very large frequency range.

2. Fundamentals of measurement in terahertz time-domain spectroscopy by Withawat Withayachumnankul from University of Adelaide in Australia and Mira Naftaly from National Physical Laboratory, the NMI in UK, surveys the metrological aspects of THz time-domain spectroscopy ranging from inadequacies of the setup and their corresponding contributions to measurement uncertainty and linearity.

3. Data Extraction from Terahertz Time Domain Spectroscopy Measurements by Maik Scheller from University of Arizona in Tucson, USA, reviews the fundamentals of data extraction techniques in $\mathrm{THz}$ time domain spectroscopy.

T. Kleine-Ostmann $(\bowtie)$

Physikalisch-Technische Bundesnastalt (PTB), Bundesallee 100, 38116 Braunschweig, Germany

e-mail: thomas.kleine-ostmann@ptb.de 
4. A Terahertz Blackbody Radiation Standard Based on Emissivity Measurements and a Monte-Carlo Simulation by Christian Monte, Bernd Gutschwager, Albert Adibekyan and Jörg Hollandt, all with the Physikalisch-Technische Bundesanstalt in Berlin, describes the source based radiometry approach for detector calibration together with an uncertainty analysis based on Monte-Carlo simulations.

5. Novel detector for traceable THz power measurements by Ralf Müller, Matthias Kehrt, Christian Monte, Andreas Steiger from the Physikalisch-Technische Bundesanstalt in Berlin and Werner Bohmeyer and Karsten Lange from the company Sensor- und Lasertechnik (SLT) in Neuenhagen, Germany, presents a new type of pyroelectric detector that may help to disseminate the THz power to other laboratories more easily in the future.

6. On the Necessity of Standardization for Sensitivity Measurements of Terahertz Cameras by Naoki Oda and Tsutomu Ishi from NEC, Iwao Hosako and Norihiko Sekine from NICT, and Hiroaki Minamide and Chiko Otani from RIKEN, all in Japan, discusses the most recent advances in the characterization of $\mathrm{THz}$ cameras.

7. Characterizing the beam properties of terahertz quantum-cascade lasers by Heiko Richter, Nick Rothbart, and Heinz-Wilhelm Hübers from the German Aerospace Center (DLR) and Technische Universität Berlin describes the methodology to characterize the beam properties of quantum cascade lasers with regard to power, beam propagation and wavefront as required for almost all applications of these and similar sources, e.g. as local oscillator.

The guest editor would like to thank all authors for their excellent contributions as well as many reviewers from all over the world for their work.

Braunschweig, May 2014

Thomas Kleine-Ostmann 